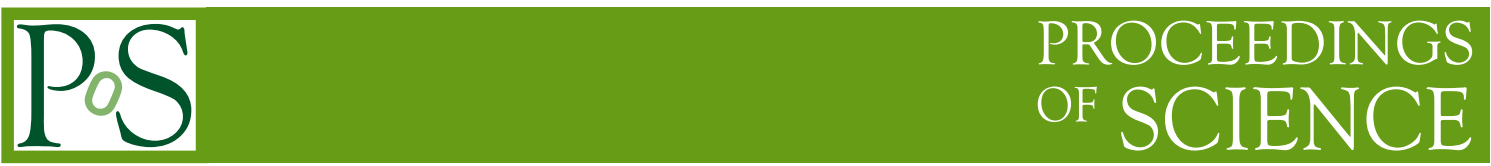

\title{
Development and Test of a Micro-Pattern Resistive Plate Detector
}

\author{
Paolo lengo ${ }^{a, *}$ \\ ${ }^{a}$ CERN, \\ Esplanade des Particules 1, 1211 Meyrin, Geneva, Switzerland \\ E-mail: paolo.iengo@cern.ch
}

We present the design and preliminary tests of a resistive strip device built with techniques developed for micro-pattern gaseous detectors. It consists of two equal electrode plates made of FR4 substrate with $250 \mathrm{Cu}$ readout strips. A $50 \mu \mathrm{m}$ insulating foil, carrying resistive lines, is glued on top of the substrate. Both the $\mathrm{Cu}$ and the resistive strips have a pitch of $400 \mu \mathrm{m}$ and width of $300 \mu \mathrm{m}$. The plates are spaced by a $2 \mathrm{~mm}$ gap and rotated by $90^{\circ}$ providing $2 \mathrm{D}$ tracking capability. With such a device the surface resistivity can be tuned to values below the ones of existing RPC (either glass or phenolic-melamine). The thin separation layer between the electrodes and the readout strips provides a better capacitive coupling of the signal, allowing to operate the detector at lower gain. Moreover, the strip-shaped resistive pattern reduces the induced charge size in the direction perpendicular to the strips. All these features go in the direction of improving the rate capability. The basic concept of this new device will be presented together with results of preliminary tests ongoing at CERN.

40th International Conference on High Energy physics - ICHEP2020

July 28 - August 6, 2020

Prague, Czech Republic (virtual meeting)

\footnotetext{
${ }^{*}$ Speaker
} 


\section{Introduction}

The development of Resistive Plate Chambers (RPC) with lower resistivity is one of the most interesting research lines in view of extending the rate capability of these devices [1]. The correlation between the bulk resistivity $\rho$ of the resistive plates and the rate capability is well known and can be written as [2]:

$$
\text { Rate Capability } \sim \frac{1}{\rho Q d}
$$

where $\mathrm{Q}$ and $\mathrm{d}$ are the average charge and the thickness of the resistive plates, respectively. Standard, single gad, phenolic-melamine (form now on called bakelite) RPC [3], with typical resistivity of $\mathrm{O}\left(10^{10} \Omega \mathrm{cm}\right)$ can efficiently work up to a rate of several $\mathrm{kHz} / \mathrm{cm}^{2}$. Possible drawbacks in increasing the rate capability by reducing the bulk resistivity value are: increase of the noise, increase of operating currents, increase on formation rate of pollutants.

Here we present a resistive plate device (RPC-like detector) built with techniques developed for micro-pattern gaseous detectors (MPGD), aiming at reaching similar performance as standard bakelite RPC in terms of time resolution and being able to efficiently work at higher particle rates.

Micro-patterning is nowadays a well established technique to build a large variety of gaseous detectors, including GEM, Micromegas, $\mu$ RWELL etc. With the introduction of a resistive layer, pioneered by the ATLAS Micromegas [4], the intensity of discharges in MPGD devices can be largely suppressed, allowing the use of such detectors in large experiments. Normally MPGD detectors are used as tracking device in applications where the particle rate is not sustainable for wire chambers; the single-layer time resolution being limited to $\mathrm{O}(10 \mathrm{~ns})$ as far as the pure gas behaviour is considered.

\section{Detector layout}

The first prototype consists of two equal electrode plates made of $2 \mathrm{~mm}$ thick fibre-glass (FR4) substrate with $17 \mu \mathrm{m} \mathrm{Cu}$ layer from which readout strips, with a pitch of $400 \mu \mathrm{m}$ and a width of $300 \mu \mathrm{m}$, are obtained by etching. A $50 \mu \mathrm{m}$ insulating foil (Kapton) is glued on top of the readout strip, carrying resistive strips with the same dimensions as the $\mathrm{Cu}$ ones (Fig. 1 left). The design is inspired by the layout of the Micromegas anode boards for the ATLAS experiment. The plates have been built at the CERN PCB workshop.

High voltage is applied to the resistive strip layer. The connection between the HV line and the resistive strip is implemented with two vias in the insulating foil, filled with Ag-based epoxy paste. The two plates are separated by a $2 \mathrm{~mm}$ gap, defined by a squared FR 4 frame, and are rotated by $90^{\circ}$ with respect to each other, providing a 2D tracking capability. The detector has 250 readout strips for each view, covering an active area of $10 \times 10 \mathrm{~cm}^{2}$. The strips are connected to two Panasonic connectors, 128 channel each, for signal readout. Gas tightness is given by an o-ring squeezed between the two detector plates.

Figure 1 shows a sketch of the board main components (left) and a picture of a single board of the resistive strip detector (right). 


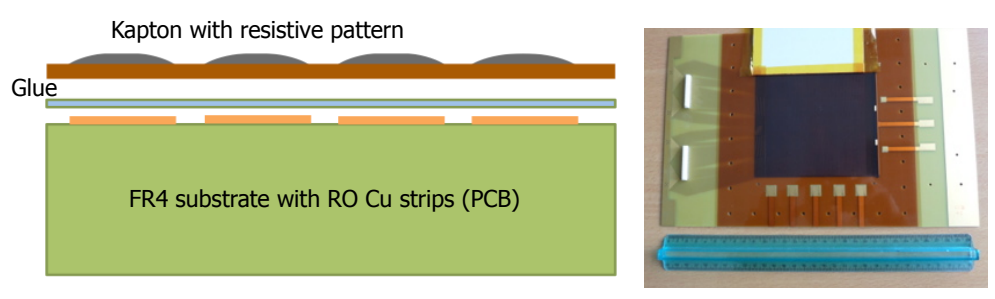

Figure 1: Sketch (not to scale) of a cross-section (left) and picture (right) of a single board of the resistive strip detector.

\section{Resistive characteristics}

Resistive strips are screen-printed on the insulating layer. The cross-section has a D-shape with a maximum height of about $15 \mu \mathrm{m}$. They are interconnected in pairs every $\mathrm{cm}$, with an alternate pattern, in order to guarantee a uniform distribution of the HV and of charge evacuation during detector operation. A mechanical polishing procedure is performed on the surface in order to smooth possible defects and imperfections of the resistive strip surface.

Figure 2 shows the resistive strip pattern, with different levels of magnification.

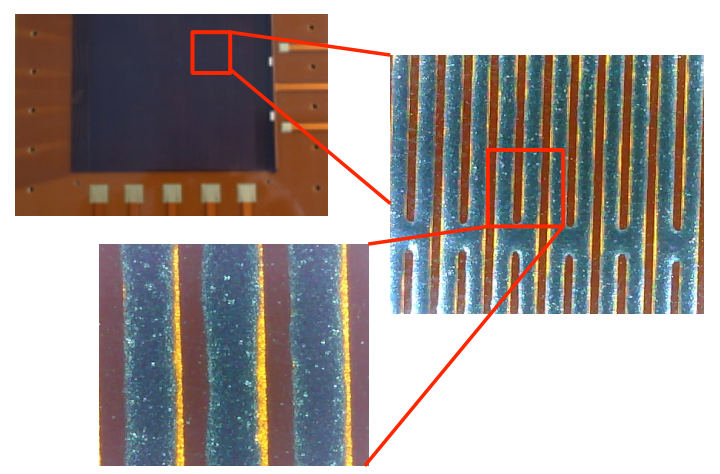

Figure 2: Pictures of the detector surface showing the parallel resistive strips. The yellowish lines are $\mathrm{Cu}$ readout strips partially visible below the resistive ones (separated by semi-transparent insulating layer). On the right picture a detail of the interconnection between resistive strips is also shown.

In traditional bakelite RPC the relevant parameter for the avalanche quenching mechanism is the bulk resistivity of the plates, as the conductive electrode is placed on the back side of the plate with respect to the side facing the gas volume. In our device the resistive strips, facing the gas volume, are directly connected to the power supply. The charge evacuation takes place along the strips: the relevant parameter is then the surface resistivity. This fact also implies a different voltage drop between the two devices: it is uniform along the surface for the RPC, while it increases with the distance from the HV supply point for the resistive strip detector.

Another important difference is related to the charge carriers. In bakelite RPC the carriers are ions, inducing the well known depletion effect ${ }^{1}$ [5]; while in our case the charge carriers are electrons and no depletion effect is expected, as was measured by dedicated studies [6] on a similar resistive insulating foil. Moreover, very limited dependency of the resistivity of the foil with temperature

\footnotetext{
${ }^{1}$ The depletion effect can be limited and even recovered by adding a controlled amount of water in the gas mixture.
} 
and humidity was measured, making this layer very robust against performance degradation during operation.

The surface resistivity has been measured with a $1 \mathrm{~cm}^{2}$ probe with respect the $\mathrm{HV}$ supply point and is shown in fig. 3 for the two plates. The measured resistivity shows a good homogeneity over the full detector surface, of the order of $0.5 \times 10^{7} \Omega /$ square. However both plates show a slightly higher resistivity on one side.
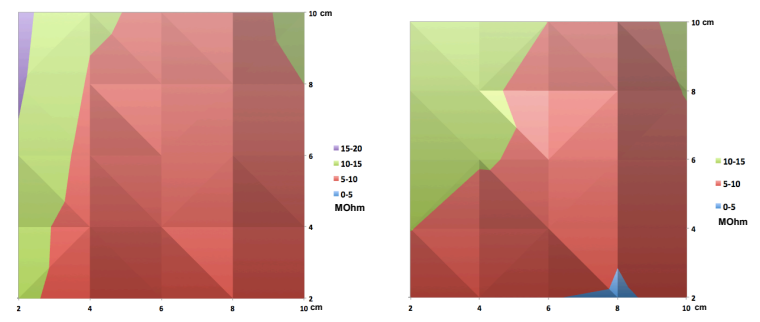

Figure 3: Resistance map (in $\mathrm{M} \Omega$ ) measured with a $1 \mathrm{~cm}^{2}$ probe with respect to the high voltage supply point, for the two plates. The HV supply line is positioned at the bottom of the plots, for both plates.

Figure 4 left shows the resistance measured with a $1 \mathrm{~cm}^{2}$ probe with respect the HV supply point as function of the distance from the HV input (along the resistive strips) for one of the two plates. The measured linearity is satisfactory. Figure 4 right shows the resistance (in $M \Omega$ ) measured with two $1 \mathrm{~cm}^{2}$ probes, at a distance of $1 \mathrm{~cm}$ from each other, as function of the distance from the HV input (along the resistive strips) for one of the two plates.
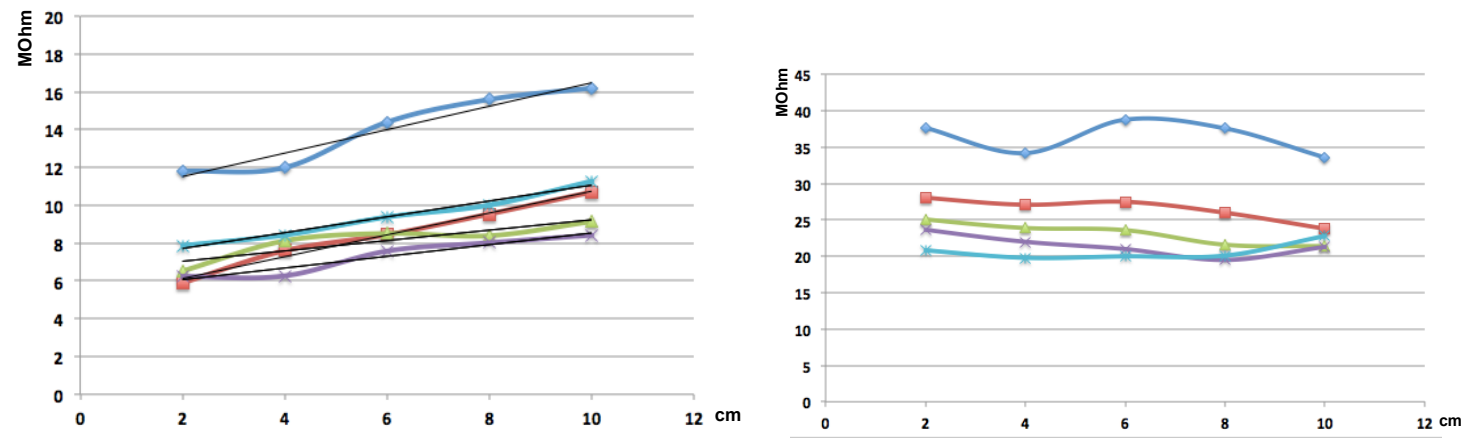

Figure 4: Left: resistance (in $\mathrm{M} \Omega$ ) measured with a $1 \mathrm{~cm}^{2}$ probe with respect to the high voltage supply as function of the distance from the $\mathrm{HV}$ input, for one plate. Right: resistance (in $\mathrm{M} \Omega$ ) measured with two 1 $\mathrm{cm}^{2}$ probes, at a distance of $1 \mathrm{~cm}$, with respect to with respect to each other, for one plate.

\section{Advantages}

The potential advantages of such a device are several:

- the possibility to tune the surface resistivity to a target value depending on the desired rate capability, while preserving the discharge quenching mechanism.

- the thin separation layer between the resistive electrodes and the copper readout strips $(<100$ $\mu \mathrm{m}$, to be compared with the typical distance of 1-2 mm for RPC) provides a better coupling of the signal, allowing to operate the detector at reduced gain; 
- the strip-shaped resistive pattern reduces the spread of the induced charge in the direction perpendicular to the strips;

All these features go in the direction of possibly improving the rate capability with respect to standard RPC.

\section{Preliminary results}

The detector was preliminary tested at CERN with the same gas mixture used for bakelite RPC for LHC experiments $\left(\mathrm{C}_{2} \mathrm{H}_{2} \mathrm{~F}_{4}: \mathrm{i}-\mathrm{C}_{4} \mathrm{H}_{10}: \mathrm{SF}_{6}\right.$ 96.2:2.5:0.3), detailed characterisation studies are planned for the next future.

Preliminary results are encouraging as the detector can be operated with good efficiency, as shown in fig. 5. The efficiency was measured with cosmics, using scintillators for triggering. 128 readout strips (corresponding to one Panasonic connector and covering half of the detector surface) were connected altogether to a single oscilloscope channel through a charge-sensitive pre-amplifier. The efficiency reached a value of $90 \%$ at a voltage of about $9000 \mathrm{~V}$, corresponding to an amplification field of $\approx 45 \mathrm{kV} / \mathrm{cm}$.

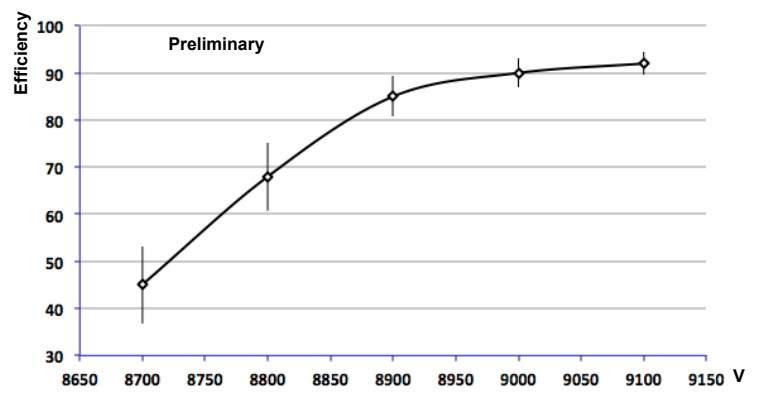

Figure 5: Preliminary measurement of the detector efficiency as function of the applied voltage.

The detector, however, showed some instabilities, including large sparks at higher voltages. This behaviour could indicate that the values of resistivity is not enough on the full detector surface to effectively quench the amplification of highly ionising events. Moreover, some small local defects have been found on the detector structure, after reopening it. These defects produce points where the amplification electrical field is more intense, more prone to sparks.

An optical inspection of the resistive surface did not show any sign of disruptive discharges ${ }^{2}$, indicating that the detector did not suffer permanent damages.

\section{Outlook}

Several optimisation are being considered. The first one is to increase the resistivity of the plates, meaning the construction of new prototypes. The latter includes the test of diamond-like

\footnotetext{
${ }^{2}$ Intense discharges can locally vaporise the carbon component of the resistive strips and leave traces of titanium oxide. These points are easily visible, even by naked eyes, as withe spots on the otherwise black surface of the resistive strips.
} 
carbon [7], (obtained with vacuum deposition of carbon) resistive layer instead of screen-printed strips, which is known to offer a smoother and more uniform surface.

Mechanical improvement to the detector structure are also considered. In order to limit the deformation of the two detector plates, subject to the large reciprocal electrostatic attraction, the FR4 frame defining the gas gap was designed with a central cross, $1 \mathrm{~mm}$ wide, sitting in the active area of the detector. The central cross was covered with Kapton tape, preventing fibre-glass filaments from the FR4 inducing discharges during the detector operations. The design of the spacer frame was, however, not optimal. It will be improved by replacing the central cross with a central spacer of more suitable material (peek) and by stiffening more the detector plates.

Optimisation of the gas composition is also an option, not explored yet at the moment.

Finally, it must be said that the resistive surface did not undergo any treatment with oil (a procedure normally used for bakelite RPC, essential to guarantee stable detector operations). A test with linseed oil is foreseen to study the impact of such treatment on the proposed detector.

\section{Conclusions}

We have built a resistive parallel plate detector with micro-pattern technique. It potentially offers several advantages to be used as trigger device in high rate environment.

The construction procedure has been demonstrated to be quite simple and the obtained resistive characteristics of the first prototype showed a good uniformity.

Preliminary results are encouraging. Performance studies are ongoing and a number of improvements are already considered, as the optimisation of the resistive layer, an improved design, optimisation of the gas mixture and surface treatment.

\section{Acknowledgment}

The author is thankful to the CERN MPT workshop team and in particular to his coordinator, Dr. Rui De Oliveira, for the construction of the first prototype and the many useful discussions.

\section{References}

[1] See for example: T. Dai et al. 2014 JINST 9 C11013

[2] M. Affatigato et al. arXiv:1507.06968

[3] R. Santonico and R. Cardarelli, Nucl. Instrum. Meth. A 187 (1981) 377

[4] The ATLAS Collaboration, CERN-LHCC-2013-006

[5] G. Aielli et al. IEEE Trans.Nucl.Sci. 53 (2006) 567-571

[6] O. Sidiropoulou, Ph.D. Thesis, CERN-THESIS-2018-140

[7] G. Bencivenni et al. Nucl.Instrum.Meth. A886 (2018) 36-39 